\title{
Jungenlungen im Nachteil bei Asthma
}

\author{
Das Risiko, als Kind ein Asthma bronchiale zu entwickeln, ist auch \\ abhängig vom Geschlecht. Welche Unterschiede hier bestehen und \\ ob präventive Maßnahmen verschieden wirken, war Thema einer \\ aktuellen Untersuchung.
}

n einer niederländischen Studie wurden Daten von 222 Kleinkindern im Alter bis zu zwei Jahren ausgewertet. Die 104 Mädchen und 118 Jungen stammten alle aus Familien mit Asthmaerkrankungen. Die Eltern erhielten kurz vor der Geburt Instruktionen zur Prävention: Sie sollten durch hygienische Maßnahmen und Encasings die Menge der Hausstaubmilben reduzieren, ihre Kinder nicht mit Zigarettenrauch belasten und die Allergenexposition durch Haustiere vermeiden. Zusätzlich wurde den Müttern empfohlen, ihre Kinder mindestens ein halbes Jahr zu stillen bzw. im Bedarfsfall hypoaller- gene Formula zu verabreichen. Nach sechs Monaten sowie einem und zwei Jahren wurden die Eltern befragt, welche Maßnahmen sie durchgeführt hatten und welche Diagnosen bei den Kindern gestellt worden waren.

Innerhalb des Beobachtungszeitraums von zwei Jahren entwickelten Jungen signifikant häufiger als Mädchen ein Asthma bronchiale (32\% vs. $18 \%)$ und ein atopisches Ekzem (36\% vs. $17 \%)$. Jungen mussten häufiger den behandelnden Ärzten vorgestellt werden. Symptome, wie Wheezing oder Kurzatmigkeit, waren bei Jungen ebenfalls signifikant häufiger.

\section{Keime sind Wegbereiter für Asthma}

\section{Welchen Einfluss hat die frühe bakterielle Besiedelung der Atem- wege auf die spätere Entwicklung eines Asthma bronchiale? Pädiater untersuchten dazu Kinder mit hohem Asthmarisiko.}

Eis ne Arbeitsgruppe um Hans Bisgaard von der Universität in Kopenhagen entnahm bei insgesamt 321 gesunden Neugeborenen im Alter von einem Monat Proben aus der Hypopharynxregion. Sämtliche Kinder wiesen ein erhöhtes Risiko für die Entwicklung einer bronchialen Obstruktion auf, da ihre Mütter unter Asthma bronchiale litten.

Die Aspirate wurden inkubiert und die angefertigten Kulturen auf Streptococcus pneumoniae, Haemophilus influenzae, Moraxella catarrhalis und Staphylococcus aureus untersucht. Die Eltern der Kinder führten in den folgenden fünf Jahren Tagebuch und notierten, ob bzw. wie häufig bei ihrem Kind Wheezing auftrat. Zusätzlich erfolgten Laboruntersuchungen sowie eine Lungenfunktionsdiagnostik.

Die bakteriologische Untersuchung zeigte bei jeweils 9\% der Proben $S$. pneumoniae und $H$. influenzae. In $8 \%$ der Fälle war $M$. catarrhalis nachweisbar. Über 60\% der Aspirate wiesen $S$. aureus auf. Die frühe Besiedlung des Hypopharynx mit den Keimen - ausgenommen $S$. aureus - war statistisch si-

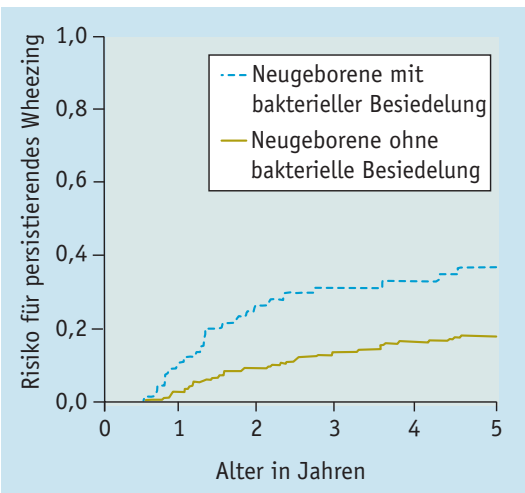

Eine Besiedelung mit bestimmten Keimen korreliert mit einer signifikant erhöhten Rate an persistierendem Wheezing.
Die Präventionsmaßnahmen wurden bei männlichen und weiblichen Kindern in etwa gleich umgesetzt. Einzige Ausnahme: Mädchen wurden wenn auch nur mit statistischem Trend - länger gestillt als Jungen. Trotz dieses Unterschieds konnte eine Regressionsanalyse signifikant belegen, dass vier Wochen längeres Stillen bei Jungen zu einer Reduktion der Episoden mit Wheezing um 13\% und derjenigen mit Kurzatmigkeit um $18 \%$ führte.

Fazit: Jungen im Alter bis zwei Jahren mit positiver Asthma-Familiennamnese entwickelten in dieser Studie deutlich häufiger ein Asthma bronchiale als Mädchen. Im Gegensatz zu Mädchen profitierten sie außerdem anscheinend von einer längeren Stillzeit.

Van Merode T et al. Gender-specific differences in the prevention of asthmalike symptoms in high-risk infants. Pediatr Allergy Immunol 2007; 18: 196-200

gnifikant mit einer Erhöhung der Atemwegserkrankungen mit Wheezing verbunden: Das Risiko für persistierendes Wheezing lag 2,4-mal höher als bei Kindern ohne diese Keime. Für akute Exazerbationen war die Wahrscheinlichkeit um etwa den Faktor 3 und für Krankenhausaufnahmen wegen Wheezing um den Faktor 3,85 erhöht.

Bei Kindern mit Besiedelung durch diese drei Keime lagen vier Jahre später die Zahl der Eosinophilen und das Gesamt-IgE, nicht aber das spezifische IgE signirifikant höher. Waren bei den Neugeborenen diese Keime nachgewiesen worden, diagnostizierten die Ärzte fünf Jahre später bei einem Drittel ein Asthma bronchiale, ohne diese Bakterien lag die Asthmaprävalenz bei $10 \%$.

Fazit: Die Besiedelung der Atemwege mit bestimmten bakteriellen Keimen im ersten Lebensmonat ist mit einem erhöhten Risiko für Wheezing und Asthma in der frühen Kindheit assoziiert. af

Bisgaard $\mathrm{H}$ et al. Childhood asthma after bacterial colonization of the airway in neonates. N Engl J Med 2007; 357: 1487-95 